\title{
OPTICAL LUMINOSITY OF ACTIVE GALACTIC NUCLEI AND THE INTENSITY OF ITS HARD RADIATION IN THE FORM OF PARTICLES AND QUANTA
}

\author{
D. N. Doikov ${ }^{1}$, A. V. Yushchenko ${ }^{2}$ \\ ${ }^{1}$ Department of mathematics, physics, and astronomy, \\ Odessa National Maritime University, Odessa, 65001, Ukraine doikov@mail.bg \\ ${ }^{2}$ Astrocamp contents research institute, Goyang, 10329, Republic of Korea \\ avyushchenko@gmail.com
}

\begin{abstract}
In this paper, we determine the causes of correlations between the observed logarithmic luminosity $\log L$ of galaxies with active nuclei (AGN) with particular attention to BL Lac type objects. The properties of flux variations of these galaxies in $\gamma$-rays $\left(\log L_{\gamma}\right)$ and in optics $\left(\log L_{O}\right)$ are associated with the combined action of cosmic rays (CR), and also with hard radiation around the entire perimeter of AGN. The necessity of using positron spectroscopy in such objects is found. The importance of high-precision measurements of $1.022 \mathrm{MeV}$ line arising from the annihilation of positrons with K-electrons of multielectron atoms in the interstellar medium of disk component of the studied galaxies is emphasized. The relationship between the single-photon positron annihilation and the Doppler line broadening of the same atoms in optics is investigated in AGN. We found that indirect evidence of the presence of a sufficient number of positrons and their annihilation are the cascade channels observed in the form of $\Delta$-resonance. According to the available data from the AGN's catalogs the calibration of their luminosity in other spectral regions is proposed.
\end{abstract}

АНОТАЦІЯ. У даній роботі визначені причини кореляцій між каталожними даними за логарифмічною світністю - $\log \mathrm{L}$ галактик 3 активними ядрами (AGN). Особливу увагу приділено галактикам типу BL Lac. Особливості зміни світностей цих галактик в $\gamma$ - $\left(\log \mathrm{L}_{\gamma}\right)$ та оптиці $\left(\log \mathrm{L}_{\mathrm{O}}\right)$ були пов'язані 3 наявністю комбінованої дії космічних променів (КП) та жорстких випромінювань по всьому периметру AGN, ï кількісним характеристикам. Обгрунтовано можливість використання позитронної спектроскопії в таких об'єктах. Підкреслено важливість високоточних вимірювань лінії $1.022 \mathrm{MeV}$, що виникає під час анігіляції позитронів 3 К-електронами багатоелектронних атомів міжзоряного середовища дискової складової досліджуваних галактик. Спираючись на попередні роботи авторів, продовжено дослідження зв'язку між однофотонною анігіляцією позитронів та доплеровским розширенням ліній цих же атомів в оптиці в AGN. Показано, що непрямим доказом наявності достатньої кількості позитронів та їх анігіляції є канали каскадів у вигляді $\Delta$-резонансу. За наявними даними з каталогів для AGN запропоноване калібрування їх світності в інших областях спектра.

Key words: active galactic nuclei, cosmic rays, inter stellar medium.

\section{Introduction}

The shells of supernovae and the active nuclei of galaxies exibit a significant field of hard radiation. The production of high-energy quanta and particles inside the young supernova shells is based on the intense radioactive decay of unstable isotopes with protons excess. Such a process is accompanied by the formation of positrons and $\gamma$-quanta. This is usual for $\beta$-processes. In the active nuclei of galaxies the charged high-energy particles are formed. Their movement along the magnetic power tubes ensures their delivery to the vast peripheral regions of AGN, providing them the sufficient energy to form their optically bright regions. The sizes of these regions can be as big as several kiloparsec in some galaxies.

Here after we are going to consider the relationship between the intensity of hard radiation and the optical luminosity produced by active nuclei of galaxies. Quite often, this luminosity noticeably exceeds the total luminosity of all stars in this galaxy. The calculations of the transport of various charged particles in the plane and halo of these galaxies (Moskalenko \& Strong, 1998) and the associated processes of photo-ionization balance (Osterbrock \& Ferland, 2005) allowed the qualitative estimates of the sources of excitation of optical transitions of atoms and molecules both for the plane 
and for the halo of these galaxies. According to the results presented by Osterbrock \& Ferland (2005) and Osterbrock (1989) the above mentioned mechanisms are not sufficient to produce the observed optical luminosities.

In this work, on the basis of taking into account the atomic photoelectric effect, previously obtained standard values of the source function of such media $S(E)$ and the electron energy distribution $f(E)$ (Doikov et al., 2018) we analyzed the reasons of excitation of the electronic structure of atoms and molecules. Basing on the results obtained by Doikov (2019) for scattering by dust particles with sizes of $10-20 \mathrm{~nm}$ it was concluded that the diffuse nature of interstellar dust scattering is an additional factor to increase the AGN's optical luminosity. The combined effect of ionization losses in gas-dust aggregates, over the entire AGN volume and, as a result, the formation of a radiation field with source function $S(E)$, is caused by the formation of cascade electron's transitions to the K-level. This phenomenon leads to the fact that the contribution to the excitation of optical transitions of the radiation field and of Auger electrons becomes comparable both in the absolute values of energy and also in its intensity.

After each event of a K-vacancy formation caused by an atomic photoelectric effect, several Auger electrons are thrown out by each of the atoms and each of them excitates, in particular, the optical transitions. In this case, the energy transfer coefficient of hard radiation is maximal. Thus, the determination of optical luminosity requires the information on the relationship between the total kinetic energy of hard radiation and particles and the total energy emitted in the optical range. Here after we are trying to solve this problem in several main steps.

In Section 2, the selection of objects from Fermi satellite catalogs - the so-called standard AGNs - is carried out. In this paper, attention is paid to the observed properties of BL Lac type of AGN. The hard radiation fluxes from such objects are slightly distorted by the inverse Compton effect and can be used to estimate the optical luminosity.

Section 3 uses the results of previous section to find the induced radiation field $S(E)$ and the Auger electron distribution function $f(E)$.

In Section 4 we estimate the optical luminosity of AGN and the spectroscopic properties of gas-dust conglomerates associated with the presence of hard radiation, especially in their peripheral regions.

In Conclusion section the general spectroscopic criteria for observations of important spectral lines of light elements in AGN are proposed.

The Discussion section compares the proposed methods for studying the AGN optical spectra and their relationship with the $\gamma$-spectra.

\section{The relationship between the AGN lumi-} nosity in hard and optical ranges

Numerous satellite and ground-based observations of galaxies with AGN have made possible the creation of catalogs where the energy fluxes in the gamma, x-ray, and optical ranges are collected. In particular, it is possible to investigate the correlations of the energy fluxes observed in B filter, with those at $1 \mathrm{keV}$ and $1 \mathrm{GeV}$. It is important because the indicated energies are in the spectral ranges where the main energy is released in AGN center $(1 \mathrm{GeV})$ and further transformed in the AGN's outer regions (1 keV and B-filter). The presence of synchrotron radiation energy in the indicated intervals is confirmed by the strong polarization and the additive structure of obseved flux.

Synchrotron radiation polarization measurements relate exclusively to the AGN's central part. The sensitivity of these observations is limited by the nearcentral regions of AGN, in which the density of gas and dust is much higher than in the outer regions. Due to the randomness of the structure of gas-dust conglomerates, the depolarization of radiation proceeds rather quickly at scales of several parsec within the galactic disks. Thus, the catalog data used for integrated flows at the indicated spectral intervals can be considered to be nonpolarized without the loss of generality.

The second important parameter is the observed galaxies absolute luminosity at the indicated energies. The fact is that in order to determine the redistribution of energy between $\gamma$-quanta, protons, $\alpha$-particles and the lepton component, it is necessary to solve the relativistic kinetic equation using the observed values of energy fluxes and the cross sections of reaction between the mentioned particles and quanta. The variable parameters in this case are the total energy flux and the flux of $\gamma$-quanta. Both these fluxes are determined by satellite observations.

A complete catalog of objects with the morphology of interest to us is several hundred. The homogeneous selection of BL Lac type objects is dozens of galaxies. It is sufficient for statistical sampling. To achieve the thermolization of a fully ionized hydrogen-helium plasma in the incident substance, in order to be able to fix the observed $\gamma$-quanta energies, the extremely high values of the substance density and temperature are necessary. These high values are unacceptable for the stable functioning of accretion disks around massive formations in the AGN's center.

One of the possible mechanisms to accelerate the charged particles is their acceleration due to the large difference of the electric field potentials (Blandford, 1977). An essential feature of this scenario is the possibility of the formation of a spark discharge with the emerging current of electrons and positrons parallel to the electric field. One electron (or positron) acceler- 
ated by this potential difference is capable of achieving high enough energy to form $\gamma$-radiation due to the socalled photon comptonization.

These photons, initially emitted tangentially to the electric field lines, can, after passing through the gap of the central part of the accretion disk, collide with a significant perpendicular component of the magnetic field and create an electron-positron pair. The pair should be accelerated and it can be the first step of cascade.

The physical system presumably adjusts its parameters to find sufficient number of charged particles for current creation. The next step can be the transformation of this current to jet. The existence of sufficiently intense cross-sectional electric and magnetic fields and the resulting acceleration mechanisms allows us to conclude that high-energy $\gamma$-quanta are formed in jets after the acceleration of mainly protons, $\alpha$-particles, and the lepton component. The use of relativistic kinetic equation at this stage allows the calculation of collision cross sections as it was proposed by Dermer (1986) and Gould (1982).

The overview of existing observations shows that AGNs flux has a significant, dense and energetically important part in $\gamma$-rays with $1 \mathrm{GeV}$ energies. Given the density of particles and quanta at the base of the jets, it should be expected that the inverse Compton effect is the main mechanism to transfer the energy of accelerated particles to quanta.

It means that the energy of particles is lost mainly due to the inverse Compton effect and inhibitory losses, while the energy of $\gamma$-quanta in this case increases unlimitedly (Gould, 1975). Let us limit our consideration by the jet base only. In this case the maximum energy of charged particles accelerated flow is in the region of several GeV. At these energies, we have a discontinuity in the spectral distribution of the marked type of galaxies due to $\Delta$-resonance. The probability of collisions of leptons with $\gamma$-quanta is significantly higher than for collisions with protons and $\alpha$-particles.

Nevertheless, it is important to determine the coefficient of energy transfer from leptons to low-energy $\gamma$-quanta and protons. Assuming that upon transition from the inner, thermolysed part of the accretion disk to the jet, the protons and leptons have an upper limit of kinetic energy no more than a few $\mathrm{MeV}$ (with a possible scattering of the order of megavolts).

Let us consider the collision cross sections for reactions between the accelerated leptons and the $\gamma$-quanta (inverse Compton effect) as well as between the accelerated leptons and the protons (taking into account the bremsstruhlung loss). The second type of declelaration is attributed to the lepton-hadron model of the interaction of protons and $\alpha$-particles with relativistic lepton.

Both types of interactions are well known in cosmic ray physics and are well studied experimentally. As- suming that in a jet the maximun of electrons energy distribution shifts toward higher energies with the increasing distance from the center, we determine the effect of the lepton current on the energy characteritics of protons and quanta. The collision of a highenergy lepton with a photon inside the jet is given by the Klein-Nishina formula. For the kinetic energy of the electron $\mathrm{E}=\gamma m c^{2}$ and the photon energy $h \nu$ according to (Lang, 1974) we have:

$$
\begin{aligned}
\sigma_{C}= & \frac{3}{4} \sigma_{T}\left\{\frac{1+q}{q^{2}}\left[\frac{2 q(1+q)}{1+2 q}-\ln (1+2 q)\right]+\right. \\
& \left.+\frac{1}{2 q} \ln (1+2 q)-\frac{1+3 q}{(1+2 q)^{2}}\right\}
\end{aligned}
$$

In the energy range of interest to us, the frequency of the radiation $\nu_{0}$ emitted as a result of the inverse Compton effect is represented by the relation $\nu_{0}=\gamma m c^{2} / h$. The parameter $\mathrm{q}$ is represented by the relation: $q=\gamma h \nu /\left(m c^{2}\right) . \sigma_{T}=6.65 \cdot 10^{-25} \mathrm{~cm}^{2}$ is the Thomson scattering cross section.

The presence of relativistic electrons and a magnetic field at the jet's base leads to the synchrotron selfcompton effect and results in a rapid energy increase of the $\gamma$-quants of the field from $6 \mathrm{MeV}$ to $1 \mathrm{GeV}$. Such an effect leads to the fact that the energies of relativistic electrons and quanta are equalized. As early as the 1950s, the rathe rhigh accuracy experiments on the scattering of fast electrons by nucleons, and the elastic character of interactions for energies less than 2-3 GeV was noticed.

Therefore, the fraction of the energy transferred from the fast electron to the proton is determined by the differential cross section $d \sigma / d \Omega_{M}$ of the Mott scattering, multiplied by a function that depends on the charge distribution inside the proton - $F(q)$ (called the form factor) and the spins of the interacting particles.

$$
\frac{d \sigma}{d \Omega}=\frac{d \sigma}{d \Omega_{M}}[F(q)]^{2}, \quad \frac{d \sigma}{d \Omega_{M}}=\frac{Z^{2} e^{4} \cos ^{2}\left(\frac{\theta}{2}\right)}{4 E^{2} \sin ^{4}\left(\frac{\theta}{2}\right)}
$$

In the interval of the energies under consideration, instead of the scattering angle $\theta$, the Breit scattering angle $-\theta_{B}$ is used according to the formula:

$$
\operatorname{ctg}^{2} \frac{\theta_{b}}{2}=\operatorname{ctg}^{2} \frac{\theta}{2} \cdot \frac{1}{1+\tau}, \quad \tau=\frac{-q^{2}}{4 M^{2}}
$$

In high-energy physics, the quantity $-q^{2}$ has the dimension $(\mathrm{GeV})^{2}$. In the present problem $-q^{2} \leq$ $3(\mathrm{GeV})^{2}$. And the $q$ value itself can be interpreted as the transmitted momentum. The value of the transmitted impulse, expressed in terms of the value of the incident impulse $p_{0}$ in the Rosenblatt formula, is expressed by the ratio:

$$
q=\frac{2 p_{0} \sin \left(\frac{\theta}{2}\right)}{\left[1+\left(\frac{2 E_{0}}{M}\right) \sin ^{2}\left(\frac{\theta}{2}\right)\right]^{1 / 2}}
$$


The various mechanisms of protons and $\alpha$-particles acceleration that occur in the AGN's central part provide such an acceleration of cosmic rays that their energy is sufficient to be detected by modern satellites. During the quiet phase intervals the AGN center should produce a CR luminosity of the order of $10^{44}-10^{46} \mathrm{erg} / \mathrm{s}$.

This energy is redistributed between the lepton and hadron components. For the occurrence of spallation reactions in the peripheral regions of AGN, a significant part of hadrons with energies of 3 or more $\mathrm{GeV}$ is necessary. The relative contents of $\alpha$-particles and protons in the AGN's center are proportional to the average ratio of helium to hydrogen. If all particles start from energies of several $\mathrm{MeV}$ and accelerate to 2-3 GeV, then during the motion from AGN through the galactic halo due to pair collisions with relativistic electrons, the momentum represented by formula (4) is transmitted to protons and $\alpha$-particles.

After the transition from accretion disk to jet, the electrons become relativistic. But it is necessary to point that the momentum transfer coefficient of the protons and $\alpha$-particles acceleration by relativistic leptons is small. Each proton should experience a minimum of three thousand collisions with such electrons on the scales of the order of $1 \mathrm{kpc}$. Let us estimate the reality of such a process analyzing the properties of observed jets in AGNs of BL Lac type objects.

The scattering of electrons by protons at low energies is Coulomb, purely elastic. For the first estimates, we take into account that the characteristic interaction radius is comparable with the De-Broglie wavelength $\lambda_{D}$. Then the minimum impact angle at which the scattering occurs is $\theta_{\min }=2 / \lambda_{D} \nu^{2}$. Then the differential scattering cross section takes the form:

$$
\sigma^{*}(v)=\frac{2 \pi}{v^{4}} \ln \frac{4}{\theta_{\min }^{2}}=\frac{2 \pi}{v^{4}} \ln \frac{2}{\left(\lambda_{D}\right)^{2} v^{4}}
$$

Collision frequency $\nu=N v \sigma(v)$. Here $v$ is the speed of the relativistic electron relative to the proton, $N$ is the concentration of protons. The actual number of atoms in a single magnetic tube with a length $L$ of $1 \mathrm{kpc}$ is $N L=10^{21}-10^{26}$ particles $/ \mathrm{cm}^{2}$. During the movement along such tubes, the number of collisions of relativistic electrons with the proton component reaches $10^{8}-10^{13}$ events, which is enough to accelerate protons and $\alpha$-particles to $\mathrm{GeV}$.

Then we can say that the particle drift along the magnetic force tubes occurs in such a way that the energy distribution function of the leptons is shifted toward several $\mathrm{MeV}$, and the maximum of the proton and $\alpha$-particle energy distribution - towards GeV. Upon reaching the galactic disk, all protons with energies above $3-5 \mathrm{GeV}$ begin to participate in inelastic scattering by hydrogen atoms, causing, among other things also the $\Delta$-resonance, according to the scheme with well known cross sections:

$\left\{\begin{array}{l}\mathrm{p}+\mathrm{H} \rightarrow \pi^{+}+\cdots ; \pi^{+} \rightarrow \mu^{+}+\cdots ; \mu^{+} \rightarrow e^{+}+\cdots \\ \mathrm{p}+\mathrm{H} \rightarrow \pi^{-}+\cdots ; \pi^{-} \rightarrow \mu^{-}+\cdots ; \mu^{-} \rightarrow e^{-}+\cdots \\ \mathrm{p}+\mathrm{H} \rightarrow \pi^{0}+\cdots ; \pi^{0} \rightarrow 2 \gamma\end{array}\right.$

It should be noted here that positrons are formed with a $50 \%$ probability (end of the first series of cascades), $25 \%$ end of the second series of cascades. And, finally, with $25 \%$ neutral pions $-\pi^{0}$ are created (Doikov et al., 2019a; Gusev et al. 2000). $\gamma$-quanta from such decays have an energy of $67.5 \mathrm{GeV}$. By varying the thickness of the galactic disk and the density of gas-dust conglomerates, we conclude that all protons, even with the cascades cross sections of $10^{-26} \mathrm{~cm}^{2}$, participate in the $\Delta$-resonance with hydrogen atoms of the interstellar medium in galactic disk with AGN.

The given observational results show one of the reasons for the correlation between luminosity in the $\gamma$ - and optical spectral ranges. In other words, $\log L_{\gamma} / \log L_{b} \approx$ const. The final products of such transformations, namely $-e^{+}, e^{-}$and the $\gamma$-quanta with energy of $67.5 \mathrm{MeV}$ are mixed with fast electrons drifting along the magnetic tubes.

Cascade transitions to K-vacancies in atoms (including the atoms in molecules) caused by ionization losses in the atoms of the galactic disk cause the luminosity of AGN's peripheral regions. In addition to quanta formed by cascade transitions, Auger electrons are injected into the medium, carrying away half the energy stored in the K-vacancy. For media with a given chemical composition, in the previous work, the energy distribution functions of Auger electrons $-F(E)$ and the source function $S(E)$ were obtained (Doikov et al., 2018).

It follows from the calculations that half of the energy of K-vacancies is emitted by atoms in the form of soft X-ray and hard UV radiation, and the rest is carried away by Auger electrons, which excite the upper atomic and molecular shells.

These mechanisms are responsible for the luminescence of both central and peripheral regions of AGN. The conversion efficiency to high-energy radiation from high-energy protons and $\alpha$-particles is $1 \%$. The efficiency reaches $40 \%$ for secondary and primary electrons and positrons,.

The correlation between the measured fluxes at 1 $\mathrm{GeV}$ energies and optical radiation, taken from the BL Lac type AGN catalog data (Fan et al., (2016) and given in Table 1, shows a stable, directly proportional correlation. An analysis of the spectroscopic properties of gas-dust conglomerates taking place in a medium penetrated by these cosmic rays was carried out by Doikov et al. (2018; 2019a,b). The comparability of Xray fluxes from the AGN center and its periphery was found. It was not expected as the mentioned fluxes are the result of independent physical mechanisms. 
Leptons, unlike hadrons in a jet, in the energy interval under consideration lose additional energy on synchrotron bremsstrahlung radiation (Gould, 1975). In this case, the observed results of $\gamma$-quanta flux measurements can be used to make preliminary estimate of the charged particles flux at the jet base, and also the X-ray (at $1 \mathrm{KeV}$ ) and optical (B-filter) fluxes. It allows estimation the spectroscopic response due to the energy transport to the AGN's peripheral regions.

Table 1 shows the observed data for AGN type BL Lac objects and our calculations for these objects. The first seven columns are taken from Fan et al. (2016). These are the designation, the redshift, the type, and the logatithms of fluxes in four spectral regions, namely in radio, optical, x-ray, and $\gamma$-ray. The last four columns are the results of our calculations and will be explained in more details in Section 4 of this paper.

The total number of BL Lac type galaxies exceeds six hundred objects. Consider, for example, the luminosity of the second galaxy in Table 1, namely J0008.0+4713. Logarithm of the total luminosity expressed in $\mathrm{erg} / \mathrm{sec}$ is $\log L_{\gamma}=44.87$. Assuming that this luminosity is caused mainly by protons, $\alpha$-particles, and leptons, we can estimate the energy and fluxes of these particles $(H)$ at the jet base using the following formula:

$$
H=L_{\gamma} / h \nu_{\gamma} \approx 6.25 \cdot 10^{61.87} \text { particles } / \text { sec } .
$$

As the average size (R) of AGN is 400 parsec, the value of peripheral flux can be written as:

$$
H_{p}=H /\left(4 p R^{2}\right) \approx 1.5 \cdot 10^{20} \text { particles } / \mathrm{cm}^{2} \cdot \mathrm{sec} .
$$

Thus, we have the upper boundary of the proton flux (not confirmed by observations). It is more realistic to use the mechanisms that occur in jets as a result of taking into account collisions of leptons $\left(e^{-}\right.$and $\left.e^{+}\right)$with protons and $\alpha$-particles proposed by Dermer (1986).

From the point of view of the relativistic kinetic theory, only the inner part of the accretion disk can be considered to be the optically thin relativistic proton plasma. In the remaining disk parts, the medium is a two-component, proton - electron plasma. Under such conditions, only the quanta with energies of not more than several $\mathrm{MeV}$ can be formed. It is the contribution to $1 \mathrm{keV}$ flux.

Bremsstrahlung manifests itself at the energies not exceeding tens of $\mathrm{MeV}$ (Gould, 1975). The formation of non-nuclear $\gamma$-quanta with several GeV energies requires the presence of strongly accelerated particles in the AGN's center, mamely at the jet base, the sufficiently high probability of collision of accelerated particles with lower-energy quanta, and also the significant magnetic field gradients.

The recent AGN observations from the Pamella and Fermi satellites found the quanta and particles of superhigh $(\mathrm{TeV})$ energies. It was shown that such an energy increase is possible only due to the inverse Compton effect between high-energy particles and quanta.
Note that the ratio of the concentration of photons, electrons and protons in the central region of AGN should differ from the concentration of protons and electrons by at least eight orders of magnitude.

Note that the choice of objects in Table 1 and the determination of the quantitative parameters of cosmic ray fluxes are required by the need of sufficiently strong magnetic fields and the intense fluxes in optics and $\gamma$ bands of the spectrum.

The motion of cosmic rays with relativistic energies in local magnetic fields leads to the appearance of synchrotron radio emission. Therefore, we will be limited to galaxies of the LB and IB type in the classification considered by Fan et al. (2016). For LB type of BL Lac objects we have the maximum spectral distribution for the frequencies $\log \nu_{p}<14$, and for IB type - in the interval $14<\log \nu_{p}<15$.

\section{Induced continuous emissions (theory)}

Thus, in the the BL Lac type galaxies with AGN we have a two-component plasma at the jet base. The first component are relativistic electrons with energy reaching several $\mathrm{GeV}$ and the second are non-relativistic protons and $\alpha$-particles with energies of the order of several MeV. Considering this physical system, we can assume that both components are in different equilibrium states. The acceleration of protons and $\alpha$-particles (with energies of several $\mathrm{MeV}$ ) by relativistic electrons (several $\mathrm{GeV}$ energies) is associated with momentum transfer from relativistic electrons minus losses due to synchrotron, bremsstrahlung, backward Compton scattering and ionization losses.

In the interval of logarithms of Lorentz factors $-1 \leq$ $\log \gamma \leq 3$ related to the physical system under consideration, the cross sections for ionization losses are prevailing at the jet base where $\log \gamma=1$. Here the electrons also achieve relativistic velocities. Then, in a halo of a BL Lac galaxy type galaxy with AGN, inside the corresponding magnetic tube, we can note the comparable values of cross sections for bremsstrahlung radiation losses due to the collision of relativistic electrons with protons and $\alpha$-particles and the cross sections describing synchrotron radiation inside magnetic tubes.

Within the framework of a given physical system, protons and $\alpha$-particles can be considered to be practically motionless. Then the rate of energy exchange between fast electrons and protons or $\alpha$-particles is (Dermer, 1986; Gould, 1982):

$$
\begin{aligned}
\frac{d u_{e}}{d t}= & -\frac{d u_{p, \alpha}}{d t}=-\frac{4 \pi e^{4}}{c} \\
& \cdot \frac{n_{p, \alpha}}{M_{p, \alpha}}\left(\ln \frac{4 \pi k T_{e}}{\omega_{p, \alpha}}+\left(-\gamma_{e}\right)\right) \cdot \frac{T_{e}-T_{p, \alpha}}{T_{e}}
\end{aligned}
$$


Table 1: BL Lac type objects: observed fluxes and relative remaining part fluxes calculated for 1 kpc distance.

\begin{tabular}{|c|c|c|c|c|c|c|c|c|c|c|}
\hline \multirow{2}{*}{ Object } & \multirow[t]{2}{*}{$\bar{Z}$} & \multirow[t]{2}{*}{ Type } & \multicolumn{4}{|c|}{ Observed fluxes } & \multicolumn{4}{|c|}{ Calculated fluxes for $1 \mathrm{kpc}$ distance } \\
\hline & & & $L_{R}$ & $L_{0}$ & $\begin{array}{c}L_{x} \\
1 \mathrm{KeV}\end{array}$ & $\begin{array}{c}L_{\gamma} \\
1 \mathrm{GeV} \\
\end{array}$ & $\mathrm{E}\left(\tau_{H}\right)$ & $\mathrm{E}\left(\tau_{H e}\right)$ & $\mathrm{E}\left(\tau_{d}\right)$ & $\mathrm{E}\left(\tau_{f}\right)$ \\
\hline 1 & 2 & 3 & 4 & 5 & 6 & 7 & 8 & 9 & 10 & 11 \\
\hline J0001.2-0748 & & IB & 42.36 & 45.39 & & 45.23 & 0.55 & 0.55 & 0.98 & 0.55 \\
\hline $\mathrm{J} 0008.0+4713$ & 0.280 & IB & 41.18 & 45.54 & 43.51 & 44.87 & 0.19 & 0.19 & 0.62 & 0.19 \\
\hline J0008.6-2340 & 0.147 & IB & 40.38 & 44.97 & 43.72 & 43.08 & 0 & 0 & 0 & 0 \\
\hline J0009.1+0630 & & LB & 42.43 & 45.04 & & 45.14 & 0.46 & 0.46 & 0.89 & 0.46 \\
\hline J0013.2-3954 & & LB & 42.74 & 44.42 & & 45.21 & 0.53 & 0.53 & 0.96 & 0.53 \\
\hline J0013.9-1853 & 0.095 & IB & 39.90 & & 43.72 & 42.88 & 0 & 0 & 0 & 0 \\
\hline J0014.0-5025 & & HB & & & 45.38 & 44.64 & 0.70 & 0.70 & 1.13 & 0.70 \\
\hline J0018.4+2947 & 0.100 & HB & 40.00 & & 43.54 & 42.84 & 0.0 & 0.0 & 0 & 0 \\
\hline J0018.9-8152 & & HB & & & 45.37 & 45.16 & 0.69 & 0.69 & 1.12 & 0.69 \\
\hline J0019.4+2021 & & LB & 43.04 & 44.42 & & 44.91 & 0.23 & 0.23 & 0.66 & 0.23 \\
\hline J0021.6-2553 & & LB & 41.88 & 45.06 & & 45.14 & 0.46 & 0.46 & 0.89 & 0.46 \\
\hline J0022.1-1855 & & IB & 41.39 & 45.60 & 44.56 & 45.13 & 0 & 0 & 0.31 & 0 \\
\hline 0022.1-5141 & & HB & & & 45.51 & 45.14 & 0.83 & 0.83 & 1.26 & 0.83 \\
\hline J0022.5+0608 & & LB & 42.57 & 44.64 & & 45.68 & 0.99 & 0.99 & 1.43 & 0.99 \\
\hline
\end{tabular}

where $\omega_{p, \alpha}$ are the plasma frequencies of protons and $\alpha$-particles. The corresponding relaxation time will be:

$$
\tau_{e, p}\left(\tau_{e, \alpha}\right)=u_{e} / \frac{d u_{e}}{d t}
$$

The acceleration time of protons and $\alpha$-particles is three orders of magnitude longer than the characteristic times of electron-electron collisions. Therefore, there are always high-energy electrons in a jet capable to transmit the momentum to protons and $\alpha$-particles. Thus, having the collision frequency of relativistic electrons with slow protons and $\alpha$-particles, we can obtain the value of the transferred energy:

$$
\left\{\begin{array}{rlrl}
\frac{d E_{r}}{d x} & \approx \frac{16}{3} N_{i} Z^{2} \frac{e^{2}}{h c}\left(\frac{e^{2}}{m c^{2}}\right)^{2} E \cdot \ln (\gamma) & & \omega>\omega_{S} \\
\frac{d E_{r}}{d x} \approx \frac{16}{3} N_{i} Z^{2} \frac{e^{2}}{h c}\left(\frac{e^{2}}{m c^{2}}\right)^{2} E \cdot \ln \left(\frac{1.4}{Z^{1 / 2}} \frac{h c}{2 \pi e^{2}}\right) & & \omega<\omega_{S}
\end{array}\right.
$$

Substituting in the first equation of the system $Z=1$, $N_{i}=1 \mathrm{~cm}^{-3}$ and the tabular data of the constants, we obtain the simple relation comfortable for further calculations:

$$
\frac{d E_{r}}{d x}=0.305 \cdot 10^{-26} E \cdot \ln (\gamma)
$$

For small values of the Lorentz factor $\gamma$-electrons can still be considered as non-relativistic $(E \leq 6 \mathrm{MeV})$, and the momentum transfer can occur mainly due to collisions. Then:

$$
\begin{aligned}
\frac{d E_{r}}{d x} & =4 \pi N Z \frac{e^{2}}{m v^{2}} \cdot \ln \left[\left(\frac{4 \pi \gamma^{2} m v^{2}}{h \omega}\right)-\left(\frac{v}{c}\right)^{2}\right] \\
& \approx 0.393 \cdot 10^{-27} \cdot \ln \left[\left(\frac{4 \pi \gamma^{2} m v^{2}}{h \omega}\right)-\left(\frac{v}{c}\right)^{2}\right] \mathrm{J} / \mathrm{cm}
\end{aligned}
$$

Formulas (10) and (11) allow us to draw the following conclusions:

1. Elastic collisions of relativistic electrons with protons and $\alpha$-particles lead to the energy losses due to bremsstrahlung and to the transfer of a part of the pulse (not more than $0.01 \%$ per collision) to accelerate these particles. The cross sections for such scattering do not exceed the thomson value $\sigma_{r}$.
2. The collisions of nonrelativistic electrons with protons and $\alpha$-particles occur in such a way that energy losses due to collisions exceed radiation losses. Formula (10) gives the correct result for the loss of electron energy up to the values of several $\mathrm{MeV}$. The accelerations of protons and $\alpha$-particles are the most effective in this case. As the electron energies increase, the transfer of mechanical energy to protons and $\alpha$-particles becomes less efficient.

3. For acceleration of protons and $\alpha$-particles to the energies of the order of $2-3 \mathrm{GeV}$ at least $10^{8}$ collisions with fast electrons are required.

\section{The optical luminosity of AGN}

In this section, we determine the optical luminosity of AGN from the observed data, namely using the columns with $L_{x}$ and $L_{\gamma}$ in Table 1 . The study of cosmic rays formed in the centers of galaxies woth various morphologies using the PAMELA satellite led to the conclusion that AGN form the particle flows with energies comparable to those generated by $\gamma$-ray fluxes. Take, for example, galaxy J0001.2-0748 of type IB with a luminosity near $L_{x}=10^{45} \mathrm{erg} / \mathrm{sec}$ at a quantum energy of $1 \mathrm{GeV}$. This value of luminosity and the presence of magnetic fields with intensities from hundreds gauss in the AGN's center to $10^{-4}$ gauss in the periphery of the magnetic tubes the particles with energies up to several $\mathrm{GeV}$ will be held .

Taking the average size of the AGN's luminous disk of the order of $0.4-1 \mathrm{kpc}$, we obtain an area of $1.44 \cdot 10^{42} \mathrm{~cm}^{2}$. For the indicated values of $L_{\gamma}$, the number of particles and quanta with an energy of 1 $\mathrm{GeV}$ is $10^{48}$ charged particles and quanta. The total luminosity of the center of our Galaxy is six orders of magnitude lower, providing on average 1 particle per $1 \mathrm{~cm}^{2}$. Therefore, for galaxy J0001.2-0748, the upper limit of the surface flux of protons, $\alpha$-particles and electrons of the order of $10^{4}$ particles $/ \mathrm{cm}^{2}$ can be considered to be acceptable. 
Let us try to find the energy distribution of these particles. The electrons have energies no higher than a few MeV. Protons and $\alpha$-particles - no higner than 2-3 $\mathrm{GeV}$. The fractional parts of electrons and protons are equal; the part of $\alpha$-particles are 10 times lower than that of protons. The thickness of the galactic disk in the region of AGN is 200-300 parsecs, which gives a thickness of absorbing media for these particles equal to $10^{21} \mathrm{~cm}$. The number of collisions of high-energy protons and $\alpha$-particles within the specified disk thickness is: $N=N_{i} N_{g} \sigma_{\Delta} L \approx 1-6$ for protons and 0.1 0.6 for $\alpha$-particles. That is, all protons and $\alpha$-particles entering the disk component experienced the transformations described by equation (6) corresponding to the channels of the $\Delta$-resonance transformation.

According to Gusev et al. (2000), the energy transfer coefficient of the initial energy in the equation (6) is $1 \%$. It means that the final energy amount is of the order of several tens of $\mathrm{MeV}$. The main mechanism of energy loss for leptons in (6) are the ionization loss, the positron annihilation, and the decay of photo-pions, as it is indicated in the third channel of (6). Ionization losses at given initial energies are caused by the formation of K-vacancies in all chemical elements or by ionization of hydrogen and helium.

The efficiency of energy transfer for the final products of equation (6) into radiation is $10-30 \%$, depending on the chemical element in which the K-vacancy is formed. In hydrogen and helium the recombination, which depends on the concentration of ions and thermal electrons, plays a large role, but the cascades instantly appear in the remaining elements, which contribute to the excitation of optical transitions. Auger electrons are formed simultaneously with cascades and lead to the same effect.

Summing up the results of the previous work (Doikov, 2019a,b) and the calculations in this article, we can show the main results in Table 1 , where the reminder of the fluxes at the distance of $1 \mathrm{kpc}$ from the center of galaxy with AGN are shown in columns from 8 to 11 .

The columns 8 and 9 show relative part of flux remaining after the absorption by only the hydrogen and only the helium. Column 10 takes into account the absorption by dust component, namely the caron and silicon dust. The last column is the part of the flux remaining after the absorption by all components, tabulated in columns 8-10. The zero values in coluns 8-11 indicate the full absorption of radiation at the distance $1 \mathrm{kpc}$ from the center. Columns 8 and 9 are similar because the results of multiplications the concentration and the cross sections for hydrogen and helium are equal.

Let us note than hydrogen and helium absorption manifests itself only in the optical component of AGN spectrum. It produces the diffuse scattering of quanta, and also the bremsstrahlung and ionizing radiation for individual recombination lines.

It is necessary to comment on the results of the AGN's luminosity calculations taking into account the presence of a dust component. In this case, columns eight and nine take into account the cascade transitions in elements such as $\mathrm{Mg}, \mathrm{Si}$, and $\mathrm{O}$ of the constituent dust. According to Doikov (2019), even in the near surface layers of dust, with a depth of 10-15 nm the above atoms form cascade transitions, with the formation of X-ray quanta. The same atoms participate in the circulation of matter of gas-dust complexes in the form of molecules and free atoms.

Of particular interest for the interpretation of radiation in x-rays are $\mathrm{Mg}$ and $\mathrm{Si}$ because their cascades $2 p-1 s, 3 d-2 p$ generate $\mathrm{x}$-ray quanta after filling a $\mathrm{K}$-vacancy near $1 \mathrm{KeV}$. Small dust particles scatter $\mathrm{x}$ ray radiation with a wavelength close to $1-10 \mathrm{~nm}$. The probability of the formation of such quanta depends on the chemical composition of the medium.

The same can be said about C, N, O elements. The mentioned process of filling $\mathrm{K}$-vacancies was studied by Doikov et al. (2018). It was found the diffuse filling of the peripheral regions of AGN with quanta of similar origin in the range from $100 \mathrm{eV}$ to $10 \mathrm{KeV}$. In this case, the observation of $\mathrm{x}$-ray flux at $1 \mathrm{KeV}$ can characterize the presence of such elements as $\mathrm{Ne}, \mathrm{Na}, \mathrm{Mg}$. But their low contents are of little use for such an analysis. The exception is Mg. Note that all elements other elements are at least six orders of magnitude less abundant.

Therefore the significant fluxes of quanta with an energy of $1 \mathrm{KeV}$ are created mainly by the central part of AGN localized by the jet base, and the diffuse component is formed by the entire volume of AGN. Formed in the AGN's peripheral parts UV and soft $\mathrm{X}$-rays are responsible for the formation of high-power optical luminescence caused by secondary radiation and Auger electrons. The cross sections for the excitation of optical transitions under the influence of a steady radiation field are at least five orders of magnitude larger than the photo-cross sections considered when K-vacancies are formed.

\section{Discussion}

The appearance of multichannel observations of AGN luminosities in various spectral regions made it possible to analyze the energy interaction of the AGN center with its periphery. At the same time, it was concluded that energy is transmitted in the form of cosmic rays and hard radiation in the form of quanta. In particular, the importance of $\Delta$-resonance is determined.

The coefficient of transformation of the initial energy of these sources into the optical radiation is determined. It is correlated with the $\gamma$-range luminosity. One of the consequences of the interaction of highenergy protons with hydrogen atoms of the interstellar 
medium is the formation of intense flux of secondary positrons.

As a result, the annihilation of secondary positrons with K-electrons gives rise to their single-photon annihilation with a $\gamma$-ray energy of $1.022 \mathrm{MeV}$ and twophoton annihilation with an energy of $0.511 \mathrm{MeV}$. The intensity of the $\gamma$-lines produced by the annihilation of positrons is a unique diagnostic spectroscopic tool for AGN's studying (Doikov et al., 2019).

The most important are the determination of the fluxes of protons and $\alpha$-particles with energies of 2-3 GeV and to the measurement of the Doppler broadening for optical lines of atoms involved in the annihilation of positrons with K-electrons.

\section{Conclusion}

The increasing amount of observations of galaxies with AGNs in various spectral ranges has expanded the range of problems to be solved and the possibility of unifying them according to measured data. In particular, the correlation between the energy fluxes in the $\gamma$-, x-ray and optical spectral regions becomes visible. Of particular interest is the correlation of the logarithms of luminosity in the $\gamma$ - and optical parts of the spectrum.

As our previous calculations showed, the formation of the AGN luminescence in the form of a $\gamma$ spectrum is associated with physical processes near a compact relativistic object, often interpreted as a massive black hole. Here, a powerful flux of cosmic rays is formed simultaneously, mainly in the form of electrons, positrons, protons and $\alpha$-particles.

In the present work, it was shown that the reason of the correlation between the $L_{\gamma}$ and $L_{B}$ luminosities is the transport of cosmic rays kinetic energy along the magnetic force tubes. These cosmic rays were formed together with $\gamma$-quanta and propagated to AGN's peripheral regions.

Cosmic rays are limited in energy by the upper limit of $3-5 \mathrm{GeV}$ since at higher energies the cosmic rays leave AGN unhindered.

We also estimated the percentage of cosmic rays energy converted into radiation as a result of ionization losses and other types of interaction of cosmic rays fluxes with gas-dust aggregates of the AGN periphery.

It amounts to about $0.5 \%-0.1 \%$ depending on the boundary conditions and the chemical composition of gas-dust conglomerates. Given the remoteness and limited information on AGN, these types of correlations allow us to estimate the expected intensity in the optical spectrum from the measured luminosity in the $\gamma$-range.

The relativistic electrons formed at the jet's base quickly lose their kinetic energy in collisions with both protons and $\alpha$-particles and, when entering the periph- eral region, are mixed with Auger electrons.

The heavy component of cosmic rays forms $\Delta$ resonance in collisions with hydrogen in gas-dust conglomerates according to the schemes shown by the equation (6). The final products of all three channels of the indicated circuit efficiently transmit energy and participate in the formation of the optical radiation field.

Acknowledgements. This work used the data from the European Space Agency (ESA) mission Gaia (https://www.cosmos.esa.int/gaia), processed by the Gaia Data Processing and Analysis Consortium (DPAC, https://www.cosmos.esa.int/web/gaia/dpac/consortium). Funding for the DPAC has been provided by national institutions, in particular the institutions participating in the Gaia Multilateral Agreement. This work was supported by the Russian Foundation for Basic Research (grant no. 18-02-00890).

\section{References}

Dermer C.D.: 1986, ApJ, 307, 47.

Blandford R.D.: 1977, MNRAS, 179, 433.

Crosas M., Weisheit J.: 1996, ApJ, 465, 659.

Doikov D.N., Andrievskii S.M., Yushchenko A.V.: 2018, Journal of Physical Studies, 22, 2901.

Doikov D.N., Yushchenko A.V., Jeong Y.: 2019a, Journal of Astronomy and Space Sciences, 36, 21.

Doikov D.N., Yushchenko A.V., Jeong Y.: 2019b, Journal of Astronomy and Space Sciences, 36, 115.

Doikov D.N.: 2019, FAS, 56, 138.

Fan J.H., Yang J.H., Liu Y. et al.: 2016, ApJS, 226, 20.

Gusev A.A., Jayanthi U.B., Martin I.M. et al.: 2000, Brazilian Journal of Physics, 50, 590.

Gould R.J.: 1982, ApJ, 254, 755.

Gould R.J.: 1975, ApJ, 196, 689.

Lang K.R.: 1974, Astrophysical Formulae, Compendium for the Physicist for Physicist and Astrophysicist. Springer (1974) (tr. Russian 1978. Mir. Moscow)

Moskalenko I.V., Strong A.W.: 1998, ApJ, 493, 694, https://doi.org/10.1086/305889.

Osterbrock D.E., Ferland G.J.: 2005, Astrophysics of gaseous nebulae and active galactic nuclei, 2ed. by D.E. G.J.Ferland. Sausalito, CA: UniversityScienceBooks, 480 p., http://adsabs.harvard.edu/abs/2006agna.book... ..OOsterbrock.

Osterbrock D.E.: 1989, Astrophysics of gaseous nebulae and active galactic nuclei, Mill Valley, CA, University Science Books, 422 p., http://adsabs.harvard.edu/abs/1989agna.book...... 